\title{
Inhalte von Regeln und Klassenmanagement
}

\section{Marie-Theres Schönbächler}

Regeln leisten einen wichtigen Beitrag zu einem erfolgreichen Unterricht, in dem wenig gestört und gut mitgearbeitet wird. Allerdings beachtete die Forschung bisher kaum, welche Inhalte in den Regeln thematisiert werden und wie die inhaltliche Gestaltung von Regeln mit dem Klassenmanagement zusammenhängt. Hier liegt der Fokus dieses Artikels. Anhand von Lehrpersonen-Daten $(N=605)$ und Schüler-Daten $(N=923)$ wird gezeigt, dass sich mit «Sozialer Interaktion» sowie "Ruhe, Ordnung» zwei Bereiche abzeichnen, bei denen ein Regelungsbedarf vorliegt. Eine einseitige Fokussierung auf Ordnungsregeln geht hingegen häufig mit einem höheren Ausmass an Unterrichtsstörungen einher. Abschliessend wird in einem Modell die indirekte Natur dieser Relation veranschaulicht und die Rolle der intermittierenden Variablen - insbesondere der Lehrperson-Schüler-Beziehung - diskutiert.

\section{Einleitung}

Klassenmanagement (im Sinne des englischen Begriffs Classroom Management und synonym verwendet zum deutschen Begriff Klassenführung) wird hier verstanden als das Handeln der Lehrperson, das auf die Errichtung und Aufrechterhaltung von Ordnungsstrukturen im Unterricht gerichtet ist und insofern vom didaktisch-methodischen direkt auf Lernprozesse gerichteten Handeln unterschieden werden kann (Doyle, 1986). Klassenmanagement hat sich in Bezug auf mehrere Aspekte als sehr bedeutungsvoll erwiesen. So haben verschiedene Studien zu effektivem Unterricht (z.B. Weinert \& Helmke, 1996; Gruehn, 2000) wie auch Meta-Analysen (Fraser, Walberg, Welch \& Hattie, 1987; Wang, Haertel \& Walberg, 1993) gezeigt, dass das Klassenmanagement einen Ansatzpunkt darstellt, über den die Lehrperson die Leistungen der Schülerinnen und Schüler beeinflussen kann. Zudem trägt eine gute Klassenführung zum Wohlbefinden der Lernenden (Eder, 2004; Hascher, 2004) wie auch zu geringerer Belastung der Lehrpersonen bei (Helmke, 2003). 
Unser Wissen darüber, was ein effektives Klassenmanagement ausmacht, beruht in wesentlichen Teilen auf amerikanischen Studien aus den 1970er bis 1990er Jahren (Doyle 1986; Emmer \& Stough, 2001). Inwiefern Regeln für ein erfolgreiches Klassenmanagement bedeutsam sind, zeigt die bekannte Beobachtungsstudie an Drittklasslehrpersonen von Emmer, Evertson und Anderson (1980). Was die effektiveren Classroom Manager in dieser Untersuchung hervorhob, war das Ausmass, in welchem Regeln und Handlungsabläufe gleich zu Beginn des Schuljahres in ein praktikables System integriert wurden und wie effektiv dieses System den Schülerinnen und Schülern gelehrt wurde. Weitere Studien bestätigten, dass erfolgreichere Lehrpersonen Klassenregeln und Handlungsabläufe im Voraus planen und vorbereiten, ihre Erwartungen gleich bei der Übernahme einer Klasse klar kommunizieren, Routinen und Handlungsabläufe etablieren, systematisch das Arbeiten und das Verhalten der Kinder beobachten sowie Feedback sowohl zu schulischen Leistungen wie auch zum Verhalten geben (Evertson \& Harris, 1992, S. 76). Der auf dieser Wissensgrundlage basierende proaktive Ansatz des Classroom Managements von Evertson und Emmer (Classroom Organization and Management Program [COMP], 2004; Emmer, Evertson \& Worsham, 2003; Evertson, Emmer \& Worsham, 2005) plädiert für die Einführung und Durchsetzung von wenigen, aber klaren, verständlichen und beobachtbaren Regeln. Er findet sich ansatzweise auch in der deutschsprachigen Ratgeberliteratur für Lehrpersonen (Nolting, 2002; Lohmann, 2003).

Im deutschsprachigen Forschungsraum wurden zwar immer wieder einzelne Aspekte des Klassenmanagements aufgegriffen, wie z.B. der Führungsstil der Lehrperson (Fend, 1998) oder die Lehrer-Schüler-Beziehung in der PISA-Studie (Moreau, Nidegger, Mariotta \& Nicoli, 2005); Untersuchungen, welche Klassenmanagement in einer umfassenden Weise analysieren, liegen allerdings nur wenige vor. Noch spärlicher ist die Befundlage, wenn nach dem Zusammenhang von Regeln und Klassenführung gefragt wird und gänzlich unberücksichtigt blieben - und dies trifft weitgehend auch auf den englischsprachigen Forschungsraum zu - Fragen, welche die Gestaltung der Regelinhalte und Konsequenzen für das Klassenmanagement betreffen. Der vorliegende Artikel soll auf der Grundlage von schweizerischen Lehrpersonen- und Schülerdaten einen Beitrag zur Schliessung dieser Lücke leisten. Es kann vermutet werden, dass nicht nur die frühe Etablierung, die Art der Einführung und die konsequente Durchsetzung eines Regelsystems mit der Klassenführung zusammenhängt, sondern auch eine inhaltliche Komponente: Welche Verhaltensweisen werden in Form von Regeln festgelegt? Mit einem explorativen Ansatz wird den Fragen nachgegangen, welche Inhalte in den Regeln gefasst werden und in welcher Relation die inhaltliche Ausrichtung der Regeln (die in einer Typen-Einteilung abgebildet wird) zum Klassenmanagement und zum Ausmass der Unterrichtsstörungen steht. 


\section{Methode, Stichprobe und Instrumente}

\section{Lehrpersonen-Stichprobe}

Im Rahmen der Studie «Klassenmanagment auf der Primarstufe» (Schönbächler, 2005) ging ein standardisierter Fragebogen zu Rahmenbedingungen des Unterrichts und mit Fragen zur eigenen Person an alle Lehrpersonen der Kantone Nidwalden, Luzern und Schwyz, welche zum Untersuchungszeitpunkt (November 2004) folgende Bedingungen erfüllten: Unterrichtstätigkeit auf der Unterstufe (1. und/oder 2. Klasse) oder auf der oberen Primarstufe (5. und/oder 6. Klasse) sowie ein Anstellungsgrad von mehr als 70 Prozent. Etwas über die Hälfte der Lehrkräfte füllte den Fragebogen aus und sendete ihn zurück. Diese Stichprobe $(\mathrm{N}=605)$ setzt sich aus 209 Lehrern und 390 Lehrerinnen (und 6 Lehrpersonen unbekannten Geschlechts), mit einem Durchschnittsalter von 35 Jahren und mit durchschnittlich 11 Jahren Unterrichtserfahrung zusammen.

\section{Schülerinnen/Schüler-Stichprobe}

Zusätzlich wurden im Kanton Schwyz, aufgeschlüsselt nach geografischer Region und nach Grösse des Schulortes, 50 Klassen der oberen Primarstufe mit Hilfe eines Zufallsverfahrens ausgewählt und 923 Schülerinnen und Schüler befragt (geschichtete Stichprobenziehung). Alle Lehrpersonen der befragten Klassen stellten sich für die Erhebung ebenfalls zur Verfügung und sind somit in der Lehrpersonen-Stichprobe integriert. Sie unterscheiden sich in den hier relevanten Variablen nicht signifikant von den anderen Lehrkräften der oberen Primarstufe und repräsentieren insofern die Stichprobe gut.

\section{Instrumente}

Zum Thema Regeln wurden neben der Regeleinführung und der Regeleinhaltung auch die Inhalte der Regeln erfasst. Lehrpersonen wie auch Schülerinnen und Schüler wurden gebeten, die drei wichtigsten Regeln im Klassenzimmer zu notieren. Zwei Raterinnen kategorisierten anschliessend unabhängig voneinander die Regelinhalte, wobei ihre Codierungen zu 95.5 Prozent übereinstimmten. Die Regeln wurden den vier Kategorien "Material, Eigentum», «Soziale Interaktion», «Ordnung, Ruhe» sowie «Zuverlässigkeit» zugeordnet (Tabelle 1). Fehlende oder nicht-zuordbare Regeln wurden als missings codiert. Für jede Person lagen schliesslich drei Codes vor, wobei einer Person auch mehrmals der gleiche Code zugewiesen werden konnte, wenn sie beispielsweise mehrere Regeln zu sozialer Interaktion aufgelistet hatte. Insgesamt lagen 1674 kategorisierte Regeln von Lehrpersonen (141 missings) sowie 2612 Regeln von Schülerinnen und Schülern (157 missings) vor. 


\section{Tabelle 1: Kategorisierung der Regelinhalte}

\begin{tabular}{|l|l|}
\hline Kategorie: & Beispiele aus den Lehrpersonendaten: \\
\hline Material, Eigentum & $\begin{array}{l}\text { Sorge tragen zu eigenem und fremdem Material. } \\
\text { - Fragen, wenn ich etwas nehme, das mir nicht gehört. }\end{array}$ \\
\hline Soziale Interaktion & $\begin{array}{l}\text { Ich höre andern zu und lasse sie ausreden. } \\
\text { Wir respektieren einander und lachen niemanden aus, wenn er es einmal } \\
\text { nicht kann. }\end{array}$ \\
\hline Ordnung, Ruhe & $\begin{array}{l}\text { Ruhig und bereit sein bei Unterrichtsbeginn. } \\
\text { Kein Herumrennen und Herumschreien. }\end{array}$ \\
\hline Zuverlässigkeit & $\begin{array}{l}\text { Wir strecken [Handzeichen geben], wenn wir etwas sagen wollen. } \\
\text { Wir arbeiten genau und sorgfältig und erledigen die (Haus)-Aufgaben } \\
\end{array}$ \\
& \begin{tabular}{l} 
gewissenhaft. \\
\hline
\end{tabular} \\
\hline
\end{tabular}

Die im Lehrerfragbogen enthaltenen Aussagen zum Klassenmanagement wurden in adaptierter Form auch den Klassen vorgelegt (Beispiel: «Ich versuche die Schülerinnen und Schüler zu verstehen» bzw. "Sie versucht, uns Schülerinnen und Schüler zu verstehen»).

Die Erhebung der Klassenführung erfolgte mit Hilfe des Linzer Diagnosebogens zur Klassenführung LDK (Mayr, Eder \& Fartacek, 2002). Die explorative Faktorenanalyse ergab die drei inhaltlich mit der Originallösung vergleichbaren Faktoren "Kontrolle» (6 Items), «Unterrichtsgestaltung» (5 Items) und «Schülerorientierung» (7 Items), die in Tabelle 2 dargestellt sind.

Die Häufigkeit der Unterrichtsstörungen, das Störausmass, wurde mit einem Einzelitem erfasst (Tabelle 2).

\section{Tabelle 2: Ausgewählte Skalen und Einzelitems zum Klassenmanagement}

\begin{tabular}{|c|c|c|c|}
\hline $\begin{array}{l}\text { Name der Skala } \\
\text { oder des Items }\end{array}$ & Beispiel-Items aus dem Lehrerfragebogen & $\begin{array}{l}\text { Kennwerte } \\
\text { Lehrpersonendaten }\end{array}$ & $\begin{array}{l}\text { Kennwerte } \\
\text { Schülerdaten }\end{array}$ \\
\hline $\begin{array}{l}\text { Klassenfüh- } \\
\text { rung1) } \\
\text { F1 Kontrolle }\end{array}$ & $\begin{array}{l}\text { Ich greife gleich ein, wenn ein Schüler oder } \\
\text { eine Schülerin zu stören anfängt. } \\
\text { - Ich achte darauf, dass die Schülerinnen und } \\
\text { Schüler im Unterricht immer beschäftigt } \\
\text { sind. }\end{array}$ & $\begin{array}{l}\text { Alpha }=.68 \\
M=3.97 \\
S=.52\end{array}$ & $\begin{array}{l}\text { Alpha }=.62 \\
M=4.08 \\
S=.57\end{array}$ \\
\hline $\begin{array}{l}\text { F2 Unterrichts } \neg \\
\text { gestaltung }\end{array}$ & $\begin{array}{l}\text { - Ich unterrichte interessant. } \\
\text { - Ich gestalte meinen Unterricht abwechslung- } \\
\text { sreich. }\end{array}$ & $\begin{array}{l}\text { Alpha }=.66 \\
\mathrm{M}=4.17 \\
\mathrm{~S}=.40\end{array}$ & $\begin{array}{l}\text { Alpha }=.80 \\
M=4.13 \\
S=.68\end{array}$ \\
\hline $\begin{array}{l}\text { F3 Schülero- } \\
\text { rientierung }\end{array}$ & $\begin{array}{l}\text { Ich interessiere mich für die persönlichen } \\
\text { Probleme und Erlebnisse der } \\
\text { Schülerinnen und Schüler. } \\
\text { - Ich lasse die Schülerinnen und Schüler vieles } \\
\text { selbst entscheiden. }\end{array}$ & $\begin{array}{l}\text { Alpha }=.66 \\
M=4.30 \\
S=.39\end{array}$ & $\begin{array}{l}\text { Alpha }=.84 \\
M=3.94 \\
S=.75\end{array}$ \\
\hline Störausmass ${ }^{2)}$ & - Gestört wird im Unterricht... & $\begin{array}{l}\text { Einzelitem } \\
M=3.24 \\
S=1.04\end{array}$ & $\begin{array}{l}\text { Einzelitem } \\
M=3.36 \\
S=1.05\end{array}$ \\
\hline
\end{tabular}

Legende: Alpha: Cronbachs Alpha, M: Mittelwert, S: Standardabweichung;

1) Ratingskala von 1, "trifft gar nicht zu» bis 5, "trifft völlig zu»;

2) Ratingskala von 1 , «sehr häufig» bis 5 , «sehr selten». 
Im Schülerfragebogen wurde der Bereich Klassenmanagement mit einer Einschätzung der Beziehung zur Lehrperson (Skala «Lehrperson-Beziehung» mit fünf Items wie «Ich mag meine Lehrerin», Alpha=.89, $\mathrm{M=3.66,S=1.01)} \mathrm{sowie} \mathrm{der} \mathrm{so-}$ zialen Bedingungen in der Klasse (Skala "Klassen-Beziehung» mit sechs Items wie "Ich fühle mich sehr wohl in unserer Klasse», Alpha=.83, M=4.08, S=.73) ergänzt.

\section{Ergebnisse}

Zunächst wird betrachtet, wie sich die aufgelisteten Regeln (drei Nennungen pro Person) insgesamt auf die vier Inhaltskategorien verteilen. Eine zweite Analyse beschäftigt sich damit, wie breit das Regelsystem einer einzelnen Lehrperson angelegt ist. Die daraus entwickelten Typen werden dann in Relation zur Klassenführung gebracht. Schliesslich wird auf der Basis der Schülerdaten mehrebenenanalytisch untersucht, wie die Typen zur Erklärung des Störausmasses beitragen.

\section{Regel inhal te}

Die Lehrerinnen und Lehrer formulierten vor allem Regeln zu «Ordnung, Ruhe» (von 80 Prozent der Stichprobe erwähnt) sowie zu «Sozialer Interaktion» (von 85 Prozent der Stichprobe erwähnt). In deutlich geringerem Ausmass fanden hingegen Aspekte wie sorgfältiger Umgang mit Material oder Zuverlässigkeit Beachtung. Weniger als 10 Prozent der Stichprobe führten Regeln zu diesen beiden Bereichen auf.

Im Folgenden wird die Häufigkeit der Nennung der einzelnen Inhaltskategorien analysiert. Verglichen werden also nicht Personen, sondern Kategorien. Bei den Regelcodes der Lehrpersonen-Stichprobe können unterrichtsstufenspezifische Differenzen konstatiert werden $\left(\mathrm{Chi}^{2}(3, \mathrm{~N}=1668)=28.75, \mathrm{p} \leq .001\right)$. Während Ordnungsregeln überproportional häufig von Unterstufen-Lehrkräften aufgelistet wurden, stammen Regeln der Kategorien «Soziale Interaktion» sowie «Zuverlässigkeit» überzufällig oft von Lehrpersonen, die eine 5. und/oder 6. Klasse unterrichten.

Vergleicht man die von den Lehrpersonen aufgeführten Regeln mit denjenigen der Schülerinnen und Schüler (Tabelle 3), fallen zwei Dinge auf: Bei Lehrenden wie Lernenden stellen «Soziale Interaktion» und "Ordnung, Ruhe» die dominanten Inhaltskategorien dar. Bei den Schülerinnen und Schülern werden jedoch Ordnungsregeln etwa doppelt so häufig genannt wie Interaktionsregeln. Die Gewichtung der Kategorien unterscheidet sich zwischen den Stichproben der Lehrenden und der Lernenden hochsignifikant $\left(\mathrm{Chi}^{2}(3, \mathrm{~N}=4286)=141.45\right.$, $\mathrm{p} \leq$.001). 
Tabelle 3: Anzahl genannte Regeln $(N=1674)$ von Lehrpersonen und Anzahl genannte Regeln $(N=2612)$ von Schülerinnen und Schülern, eingeteilt in Regelinhalts-Kategorien

\begin{tabular}{|l|r|r|r|r|}
\hline Kategorie & \multicolumn{2}{|r|}{ Von Lehrpersonen genannt } & \multicolumn{2}{|r|}{ Von Schüler/-innen genannt } \\
& $N$ & Anteil & $N$ & Anteil \\
\hline Material, Eigentum & 44 & $2.6 \%$ & 38 & $1.5 \%$ \\
\hline Soziale Interaktion & 811 & $48.4 \%$ & 818 & $31.5 \%$ \\
\hline Ordnung, Ruhe & 770 & $46.0 \%$ & 1593 & $61.6 \%$ \\
\hline Zuverlässigkeit & 49 & $2.9 \%$ & 135 & $5.3 \%$ \\
\hline Total & 1674 & $100 \%$ & 2612 & $100 \%$ \\
\hline
\end{tabular}

\section{Breite bzw. Fokussierung der Regeln}

Für die weitere Analyse ist relevant, ob die Lehrpersonen jeweils nur Regeln einer einzigen Kategorie aufgelistet haben (Fokussierung) oder ob ihr Regelsystem Regeln unterschiedlicher Inhaltskategorien umfasst (Breite). Dazu wurde jede Lehrperson einem der folgenden Typen zugeordnet: Im «Schwerpunkttyp Interaktion» sind Lehrkräfte zusammengefasst, welche ausschliesslich Interaktionsregeln auflisteten (N=91). Dem «Schwerpunkttyp Ordnung» gehören diejenigen Lehrpersonen an, die nur Ordnungsregeln notierten $(\mathrm{N}=70)$. Lehrpersonen des "gemischten Typs» ( $\mathrm{N}=415)$ nannten Regeln aus verschiedenen Kategorien. Es gab keine Lehrpersonen, welche ausschliesslich Regeln der Kategorie «Material, Eigentum» oder der Kategorie "Zuverlässigkeit» aufführten.

Gefragt wird nun, wie sich die Klassenführung dieser drei Typen unterscheidet. Eine Varianzanalyse belegt signifikante Differenzen zwischen den drei Typen bezüglich des Faktors "Schülerorientierung» $(\mathrm{F}(2,579)=3.67, \mathrm{p} \leq .05)$. Lehrpersonen des «Schwerpunkttyps Ordnung» schätzten ihre Schülerorientierung signifikant tiefer ein als Lehrkräfte des «Schwerpunkttyps Interaktion» (Scheffé-Test $\mathrm{p} \leq .05$ ) und auch tendenziell tiefer als die Lehrpersonen des "gemischten Typs». Eine strikte Ausrichtung auf Ordnungsaspekte geht demnach mit geringerem Interesse für Probleme und Erlebnisse der Kinder sowie weniger Eingehen auf deren Wünsche einher.

Da die Skala «Schülerorientierung» die Beziehung der Lehrperson zu den Kindern spiegelt, das Interesse und Vertrauen, das sie ihnen entgegenbringt, kann vermutet werden, dass sich dieser Befund auch in der Wahrnehmung der Schülerinnen und Schüler niederschlägt. In der Folge werden deshalb die Schülerdaten herangezogen.

\section{Regel-Schwerpunktsetzung und Störausmass}

Eine zentrale Variable, welche die Qualität des Unterrichts beleuchtet, ist die sowohl bei den Lehrpersonen wie auch bei den Schülerinnen und Schülern erho- 
bene Einschätzung der Störhäufigkeit im Unterricht (Tabelle 2). Im Gegensatz zu einigen anderen Variablen und Skalen, bei denen die Lehrenden ihr Klassenmanagement selbst eher positiver einschätzten als dies ihre Schülerinnen und Schüler taten (Schönbächler, 2005), liegt beim Störausmass eine hohe Übereinstimmung zwischen Lehrer- und Schülersicht vor, was T-Test $(\mathrm{t}(49)=.98$, n.s.) und Pearson-Korrelation ( $\mathrm{r}=.45, \mathrm{p} \leq .01)$ belegen. In Klassen, die das Störausmass hoch einschätzen, dürfte demnach auch die Lehrperson den Unterricht als häufig gestört wahrnehmen.

Im Folgenden soll mehrebenenanalytisch geklärt werden, ob - und allenfalls wie - sich der Regel-Schwerpunkttyp (Lehrervariable) im Störverhalten (Schülervariable) manifestiert. Dazu wurde das Programm MLwiN 2.02 (Rasbash, Steele, Browne \& Prosser 2004) eingesetzt.

In der einfachsten Form des Modells, im leeren Modell (Nullmodell), wird geschätzt, wie sich die Varianz auf die beiden Ebenen Individuum (Level 1) und Klasse (Level 2) verteilt. Dem Nullmodell in Tabelle 4 kann entnommen werden, dass sich nicht nur die Einschätzung des Störausmasses zwischen den Schülern einer Klasse (signifikante Varianz auf Level 1), sondern auch zwischen den Klassen (signifikante Varianz auf Level 2) bedeutsam unterscheidet, was eine Mehrebenenanalyse rechtfertigt. Der Varianzanteil auf Klassenebene macht etwa einen Viertel der Gesamtvarianz aus. Mit dem Zufügen von Prädiktor-Variablen wird nun versucht, ein Teil dieser Varianz aufzuklären.

Im Modell 1 (Tabelle 4) wurde der Regel-Schwerpunkttyp als Prädiktor integriert. Da es sich dabei um eine kategoriale Variable handelt, sind die Ausprägungen "SI-Typ» (nur Regeln zur Sozialen Interaktion) sowie "OR-Typ» (nur Regeln zu Ordnung, Ruhe) als Dummy-Variablen in Kontrast zum "gemischten Typ» (Regeln zu mehreren inhaltlichen Kategorien) zu interpretieren. Die Variable Regel-Schwerpunkttyp erklärt gut einen Viertel der ursprünglichen Varianz der Unterschiede zwischen den Klassen, wozu der "OR-Typ» signifikant beiträgt. Die Klassen von Lehrpersonen, die ausschliesslich Ordnungsregeln auflisteten, schätzten also Störausmass negativer (mehr Störungen im Unterricht) ein als die Klassen der anderen beiden Typen. Klassen, deren Lehrkraft nur Interaktionsregeln notierten, unterscheiden sich nicht signifikant vom "gemischten Typ». 
Tabelle 4: Mehrebenenanalytische Vorhersage des Störausmasses durch den RegelSchwerpunkttyp der Lehrperson sowie den Schüler-Variablen LehrpersonBeziehung, Klassen-Beziehung und Unterrichtsgestaltung

\begin{tabular}{|l|lll|}
\hline & $\begin{array}{l}\text { Störausmass } \\
\text { Nullmodell }\end{array}$ & $\begin{array}{l}\text { Störausmass } \\
\text { Modell 1 }\end{array}$ & $\begin{array}{l}\text { Störausmass } \\
\text { Modell 2 }\end{array}$ \\
\hline fixed part & & & \\
Intercept & $.01(.01)$ & $.13(.08)$ & $.07(.07)$ \\
SI-Typ & & $-.10(.16)$ & $-.05(.13)$ \\
OR-Typ & $-.57(.24)^{*}$ & $-.34(.21)$ \\
Lehrperson-Beziehung & & & $.14(.05)^{* *}$ \\
Klassen-Beziehung & & & $.07(.03)^{*}$ \\
Unterrichtsgestaltung & & $.16(.05)^{* * *}$ \\
random part & $.23(.06)^{* * *}$ & $.17(.04)^{* * *}$ & $.11(.03)^{* * *}$ \\
Varianz Level 2 & $.76(.04)^{* * *}$ & $.74(.04)^{* * *}$ & $.70(.04)^{* * *}$ \\
$\quad$ Varianz Level 1 & & & \\
likelihood ratio des Modells & & $2239.12^{* * *}$ & $2159.02^{* * *}$ \\
(df=100) & $2122.04^{* * *}$ & & \\
\hline
\end{tabular}

Legende: Signifikanzen: ${ }^{*} \mathrm{p} \leq .05,{ }^{* *} \mathrm{p} \leq .01,{ }^{* * *} \mathrm{p} \leq .001$; SI-Typ: Schwerpunkttyp Interaktion, OR-Typ: Schwerpunkttyp Ordnung; alle Schüler-Variablen z-standardisiert, Standardmessfehler in Klammern

Im Modell 2 wurden nun zusätzlich Schülervariablen einbezogen, die sich in vorgängig gerechneten Mehrebenenanalysen als signifikante Prädiktoren für die Erklärung des Störausmasses bewährt haben. Dabei handelt es sich um die faktorenanalytisch gewonnenen Variablen «Lehrperson-Beziehung» und "KlassenBeziehung» sowie um den Klassenführungsfaktor «Unterrichtsgestaltung» (Tabelle 2). Gemäss Tabelle 4 leisten alle drei neu integrierten Prädiktoren einen signifikanten Beitrag zur Aufklärung der Varianz. Der Einbezug führt zu einer deutlichen Abnahme der Varianz auf Klassenebene. Neu wird gut die Hälfte der ursprünglichen Unterschiede zwischen den Klassen erklärt. Allerdings zeigt der Vergleich von Modell 1 mit Modell 2 in Tabelle 4, dass der signifikante Effekt des «OR-Typs» entfällt, wenn das Modell mit relevanten Schülervariablen erweitert wird. Dieses Verschwinden des in Modell 1 zum Ausdruck kommenden Effekts des «Schwerpunkttyps Ordnung» könnte - so wird vermutet - auf einem indirekten Zusammenhang zwischen dem Regel-Schwerpunkttyp und dem Störausmass beruhen.

Um diese These zu prüfen, wurde in erneuten unabhängigen Mehrebenenanalysen der Regel-Schwerpunkttyp als Prädiktor für die abhängigen Schülervariablen «Lehrperson-Beziehung», «Klassen-Beziehung» und «Unterrichtsgestal- 
tung» gesetzt. Für die Variable "Klassen-Beziehung» ergaben sich nur geringe Varianzanteile auf Level 2 und kein bedeutsamer Erklärungsgehalt des RegelSchwerpunkttyps. Anders hingegen - so veranschaulicht Tabelle 5 - sehen die Befunde bei den Analysen für die "Lehrperson-Beziehung» (Modell 3) sowie für die «Unterrichtsgestaltung» (Modell 4) aus. Hier ist ein hoher Anteil der Varianz auf Unterschiede zwischen den Klassen zurückzuführen (36 Prozent bzw. 31 Prozent). Beides sind Bereiche, welche die Lehrperson besser beeinflussen kann als beispielsweise das Störverhalten oder die sozialen Beziehungen in der Klasse. Folglich werden Lehrperson-Beziehung und Unterrichtsgestaltung innerhalb der Klassen homogener (weniger Varianz auf Level 1) und zwischen den Klassen heterogener (mehr Varianz auf Level 2) wahrgenommen. Der «OR-Typ» leistet in beiden Modellen ( 3 und 4) einen signifikanten Erklärungsbeitrag. Bei der Lehrperson-Beziehung in Modell 3 kann ein Drittel der Unterschiede zwischen den Klassen mit der Variable Regel-Schwerpunkttyp erklärt werden; bei der Unterrichtsgestaltung in Modell 4 sind es sogar fast zwei Drittel.

Tabelle 5: Mehrebenenanalytische Vorhersage der Lehrperson-Beziehung und der Unterrichtsgestaltung (Schülervariablen) durch den Regel-Schwerpunkttyp (Lehrervariable)

\begin{tabular}{|c|c|c|c|c|}
\hline & $\begin{array}{l}\text { Lehrperson- } \\
\text { Beziehung } \\
\text { Nullmodell }\end{array}$ & $\begin{array}{l}\text { Lehrperson- } \\
\text { Beziehung } \\
\text { Modell } 3\end{array}$ & \begin{tabular}{|l|} 
Unterrichts- \\
gestaltung \\
Nullmodell
\end{tabular} & $\begin{array}{l}\text { Unterrichts- } \\
\text { gestaltung } \\
\text { Modell } 4\end{array}$ \\
\hline \multicolumn{5}{|l|}{ fixed part } \\
\hline Intercept & $.02(.09)$ & $.18(.09)$ & $.01(.08)$ & $.15(.09)$ \\
\hline SI-Typ & & $-.10(.18)$ & & $-.10(.17)$ \\
\hline OR-Typ & & $-.87(.27)^{* *}$ & & $-.54(.25)^{*}$ \\
\hline \multicolumn{5}{|l|}{ random part } \\
\hline Varianz Level 2 & $.36(.08)^{* * *}$ & $.24(.06)^{* * *}$ & $.31(.07)^{* * *}$ & $.11(.03)^{* * *}$ \\
\hline Varianz Level 1 & $.63(.03)^{* * *}$ & $.61(.03)^{* * *}$ & $.69(.03)^{* * *}$ & $.70(.04)^{* * *}$ \\
\hline likelihood ratio des Modells & & & & \\
\hline$(\mathrm{df}=100)$ & $2304.98^{* * *}$ & $2117.52^{* * *}$ & $2376.60^{* * *}$ & $2171.63^{* * *}$ \\
\hline
\end{tabular}

Legende: Signifikanzen: ${ }^{*} \mathrm{p} \leq .05,{ }^{* *} \mathrm{p} \leq .01,{ }^{* * *} \mathrm{p} \leq .001$; SI-Typ: Schwerpunkttyp Interaktion, OR-Typ: Schwerpunkttyp Ordnung; alle Schüler-Variablen z-standardisiert, Standardmessfehler in Klammern

Wie schon in Modell 1 ergibt sich auch in Modell 3 und 4 ein kritisches Bild für den «Schwerpunkttyp Ordnung»: Die Klassen dieser Lehrkräfte schätzten ihre Beziehung zur Lehrperson bedeutend negativer ein und bewerteten auch den Unterricht als weniger interessant und abwechslungsreich als die anderen Klassen.

Werden die Ergebnisse der verschiedenen Mehrebenenanalysen in der Zusammenschau betrachtet, ergibt sich das untenstehende Gesamtmodell (Abbildung 1). 


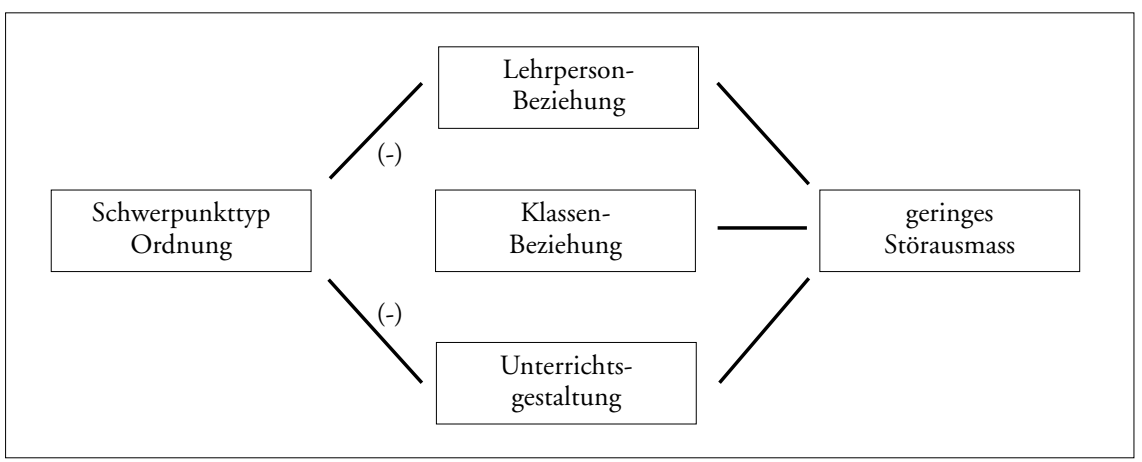

Abbildung 1: Gesamtmodell zum Regelinhalts-Schwerpunkt Ordnung und zum Ausmass des Störverhaltens

Obwohl das Gesamtmodell mehrebeneanalytisch nicht gerechnet werden kann, stützen die einzelnen Analyseschritte die Annahme, dass eine strikte inhaltliche Fokussierung der Regeln auf den Bereich Ordnung und Ruhe auf indirektem Wege, nämlich über die moderierenden Variablen Lehrperson-Beziehung und Unterrichtsführung, negativ mit dem Störausmass im Unterricht zusammenhängt.

\section{Diskussion}

Wie gezeigt wurde, spielen in den von den Lehrpersonen wie auch den Schülerinnen und Schülern aufgeführten Regeln die beiden Bereiche "Material, Eigentum» und "Zuverlässigkeit» nur eine marginale Rolle. Damit unterscheidet sich die Schweizer Stichprobe zwar von den Lehrkräften einer US-amerikanischen Untersuchung (Howard \& Norris, 1994), bei denen ein Drittel der Lehrpersonen auch Regeln zum Umgang mit Material und zur zuverlässigen Aufgabenerledigung formulierten. Doch in der amerikanischen Studie bilden ebenfalls die Interaktion, das Sprechen und das Bewegen im Klassenzimmer die meistgenannten Regelinhalte. Es scheint also kulturübergreifende Kernbereiche zu geben, welche in einem klassenmässig organisierten Unterricht geregelt werden sollten. «Soziale Interaktion» sowie «Ordnung, Ruhe» stellen solche Kernbereiche dar.

Gerade in der Gewichtung dieser letztgenannten Kategorien unterscheiden sich Lehrende und Lernende signifikant. Bei den Lehrpersonen sind beide Bereiche etwa gleich oft unter den wichtigsten Regeln berücksichtigt. Schülerinnen und Schüler notierten Regeln zu Ruhe und Ordnung jedoch doppelt so häufig wie Interaktionsregeln. Zieht man die unterschiedlichen Rollen in Betracht, lässt sich dieser Umstand gut nachvollziehen. Während die Lehrperson für die Klasse als Ganzes verantwortlich ist - was auch Interaktionen zwischen einzelnen Kin- 
dern einschliesst -, interessieren sich die Schüler bzw. Schülerinnen betreffend Regeln vorrangig dafür, was für sie selbst erlaubt oder verboten ist. Im übergreifenden Fokus der Lehrpersonen erhalten Interaktionsregeln somit grössere Bedeutung als in der enger gehaltenen Perspektive der Kinder. Die sehr ausgeprägte Gewichtung von Ordnungsregeln bei den Kindern könnte zudem auch einem Bedürfnis der Schülerinnen und Schüler entspringen: Schüleraussagen aus einer amerikanischen Studie auf der Sekundarstufe (Cothran, Kulinna \& Garrahy, 2003) stützen die Vermutung, dass Kinder einen ruhigen, ungestörten Unterricht schätzen und als hilfreich für ihr Lernen wahrnehmen.

In der an die Praktiker gerichteten Fachliteratur trifft man neben formalen Kriterien (beispielsweise der positiven Formulierung von Regeln) oft auch auf Tipps, welche die inhaltliche Breite betreffen: Regeln sollen verschiedene Unterrichtssituationen möglichst umfassend abdecken (Evertson \& Poole, 2004; Evertson Emmer \& Worsham, 2005). Hier zeigen die vorgestellten Daten, dass die inhaltliche Ausrichtung der Regeln durch die Lehrperson mit dem Störausmass der Schüler und Schülerinnen in Zusammenhang steht. Die Fokussierung auf Ordnungsregeln geht einher mit mehr Störungen im Unterricht. Von einer kausalen Interpretation muss angesichts fehlender Längsschnittdaten Abstand genommen werden. Die Zusammenhänge lassen sich aber theoretisch analysieren. Es ist einleuchtend, dass in einem häufig gestörten Unterricht für die Lehrperson die Errichtung von Ruhe und Ordnung im Vordergrund steht und sie entsprechende Regeln implementiert. Kritisch ist meines Erachtens, die konstatierte indirekte Wirkung über die Variable der Lehrperson-Beziehung. Schon die klassischen Studien zum Erziehungsstil betonen die Bedeutsamkeit einer anerkennenden, respektvollen Beziehung (Baumrind, 1991; Tausch, 1998). Wenn diese Beziehung - wie bei Herzog (2002) dargestellt - als konstitutionelle Basis für Unterricht betrachtet wird, als egalitäre Ebene, welche das asymmetrische Verhältnis von Lehren und Lernen erst tragbar macht, dann ist hier der wunde Punkt auszumachen. Eine einseitige Ausrichtung auf formale Ordnungsregeln kann von den am Unterricht Beteiligten auch als Nicht-Gewichtung des sozialen Interaktionsaspektes interpretiert werden. Damit wird die soziale Basis geschwächt, welche es erst ermöglicht, dass die Schülerinnen und Schüler «die Zumutung, die der Unterricht in didaktischer Hinsicht darstellt» (ebd., 504), hinnehmen. Eine Wechselwirkungsannahme zwischen strikten Ordnungsregeln einerseits und Störverhalten andererseits ist also durchaus plausibel.

Die vorgestellten Analysen basieren auf den Daten zufällig ausgeloster Klassen und umfassen nicht nur Schülerinnen und Schüler «erfolgreicher» Lehrpersonen (wie z.B. bei Mayr, Eder \& Fartacek, 1991), sondern auch solche, die ihre Beziehung zur Lehrkraft kritisch einschätzen. Sie tragen somit zur Erhellung des für die Unterrichtspraxis sehr bedeutsamen, aber oft ignorierten Aspektes der belasteten Lehrperson-Schüler-Beziehung bei. Gleichzeitig liefern sie Hinweise für weiterführende Untersuchungen. Möglicherweise stellen gerade die intermittierenden Variablen «Lehrperson-Beziehung» und «Unterrichtsgestaltung» Ansatz- 
punkte dar, an welchen ein ungünstiges gegenseitiges Aufschaukeln von strikten Ordnungsvorgaben durch die Lehrperson und Störverhalten durch die Schülerinnen und Schüler durchbrochen werden könnte. Dies kann jedoch auf Grund der vorliegenden Daten nicht beurteilt werden. Vertiefende Forschung wäre hier gefordert.

Ein weiterer Ansatzpunkt für weiterführende Studien kann beim Ausmass der Schülermitsprache bei der Einführung von Klassenregeln geortet werden, einem Aspekt, der in der vorliegenden Studie nicht erfasst wurde. Der Einbezug der Klassen in die Aushandlung der Regeln wird auch vom Schweizer Dachverband der Lehrerinnen und Lehrer LCH unterstützt (Szaday, Kummer, Pool \& Mettauer, 2000) und soll die Akzeptanz und Einhaltung der Regeln stärken (Evertson, Emmer \& Worsham, 2005). Noch wissen wir jedoch sehr wenig darüber, inwieweit dies tatsächlich zutrifft. Es kann aber vermutet werden, dass das «ernst nehmen» der Kinder, das sich in einer gemeinsamen Regelfestlegung manifestiert, von den Schülerinnen und Schülern als «Vertrauensvorschuss» wahrgenommen wird und quasi den ersten Schritt beim Aufbau einer guten Beziehung bildet, die wiederum bedeutsam das Klassenmanagement prägt.

\section{Literatur}

Baumrind, D. (1991). Rearing Competent Children. In W. Damon (Hrsg.), Child Development Today and Tomorrow (p. 349-378). San Francisco: Jossey-Bass.

Classroom Organization an Management Program COMP, (2004). Creating Conditions for Learning [On-line]. Peabody College, Vanderbilt University Nashville, TN. Available: [http://www.comp.org/aboutus.htm] [Dec. 20th. 2005].

Cothran, D. J., Kulinna, H. \& Garrahy, D. (2003). "This is kind of giving a secret away...»: students' perspectives on effective class management. Teaching and Teacher Education, 19, 435-444.

Doyle, W. (1986). Classroom Organization and Management. In M. C. Wittrock (Ed.), Handbook of Research on Teaching (3rd ed., p. 392-397). New York: Macmillan.

Eder, F. (2004). Der Einfluss einzelner Lehrpersonen auf das Befinden von Schülerinnen und Schüler. In T. Hascher (Hrsg.), Schule positiv erleben. Ergebnisse und Erkenntnisse zum Wohlbefinden von Schülerinnen und Schülern (S. 91-112). Bern: Haupt.

Emmer, E. T., Evertson, C. M. \& Anderson, L. M. (1980). Effective Classroom Management at the Beginning of the School Year. The Elementary School Journal, 80 (5), 219-231.

Emmer, E. T., Evertson, C. M. \& Worsham, M. E. (2003). Classroom management for secondary teachers (6th ed.). Englewood Cliffs, NJ.: Prentice Hall.

Emmer, E. T. \& Stough, L. M. (2001). Classroom Management: A Critical Part of Educational Psychology, with Implictions for Teacher Education. Educational Psychologist, 36, 103-112.

Evertson, C. M., Emmer, E. T. \& Worsham, M. E. (2005). Classroom management for elementary teachers (7th ed.). Boston: Allyn \& Bacon.

Evertson, C. M. \& Harris, A. H. (1992). What We Know About Managing Classrooms. Educational Leadership, 49 (7), 74-79.

Evertson, C. M. \& Poole, I. (2004). Norms and Expectations [On-line]. IRIS Center for Faculty Enhancement, Nashville, TN: Peabody College, Vanderbilt University. Available:[http://iris.peabody.vanderbilt.edu/casestudies.html] [Oct. 5th 2005].

Fend, H. (1998). Qualität im Bildungswesen. Schulforschung zu Systembedingungen, Schulprofilen und Lehrerleistung. Weinheim: Juventa. 
Fraser, B. J., Walberg, H. J., Welch, W. W. \& Hattie, J. A. (1987). Syntheses of Educational Productivity Research. International Journal of Educational Research, 11, 145-252.

Gruehn, S. (2000). Unterricht und schulisches Lernen: Schüler als Quellen der Unterrichtsbeschreibung. Münster: Waxmann.

Hascher, T. (2004). Woblbefinden in der Schule. Münster: Waxmann.

Helmke, A. (2003). Unterrichtsqualität - erfassen, bewerten, verbessern. Seelze: Kallmeyer.

Herzog, W. (2002). Zeitgemässe Erziehung. Die Konstruktion pädagogischer Wirklichkeit. Weilerswist: Velbrück Wissenschaft.

Howard, N. A. \& Norris, M. R. (1994). Source, Characteristics, and Percieved Effectiveness of Classroom Rules. Paper presented at the Kentucky Grow Conference. Kentucky: February 25.

Lohmann, G. (2003). Mit Schülern klarkommen. Professioneller Umgang mit Unterrichtsstörungen und Disziplinkonflikten. Berlin: Cornelsen.

Mayr, J., Eder, F. \& Fartacek, W. (1991). Mitarbeit und Störung im Unterricht: Strategien pädagogischen Handelns. Zeitschrift für Pädagogische Psychologie, 5 (1), 43-55.

Mayr, J., Eder, F. \& Fartacek, W. (2002). Linzer Diagnosebogen zur Klassenführung (LDK) [On-line]. Verfügbar unter:[http://www.padl.ac.at/staff/maj/ff11.htm] [9. Februar 2004].

Moreau, J., Nidegger, C., Mariotta, M. \& Nicoli, M. (2005). Schülerkompetenzen und ihr Kontext: Versuch einer systemischen Analyse (PISA 2003, Kompetenzen für die Zukunft: Zweiter Nationaler Bericht, S. 75-96). Neuchâtel: Bundesamt für Statistik.

Nolting, H.-P. (2002). Störungen in der Schulklasse. Ein Leitfaden zur Vorbeugung und Konfliktlösung. Weinheim: Beltz Taschenbuch.

Rasbash, J., Steele, F., Browne, W. \& Prosser, B. (2004). A User's Guide to MLwiN. London: Centre for Multilevel Modelling, Institute of Education, University of London.

Schönbächler, M.-T. (2005). Klassenmanagement auf der Primarstufe. Dokumentation zur Datenerhebung bei den Lehrpersonen und bei den Schülerinnen und Schülern (Forschungsbericht Nr. 32). Bern: Universität Bern, Institut für Pädagogik und Schulpädagogik, Abteilung Pädagogische Psychologie.

Szaday, C., Kummer, A., Pool, S. \& Mettauer, B. (2000). Diziplinschwierigkeiten gehen uns alle an! Ein Handweiser zum Umgang mit Disziplinschwierigkeiten in der Schule. Zürich: Verlag $\mathrm{LCH}$.

Tausch, R. (1998). Personzentrierte Unterrichtung und Erziehung. In D. H. Rost (Hrsg.), Handwörterbuch Pädagogische Psychologie (S. 397-403). Weinheim: Beltz, Psychologie Verlags Union.

Wang, M. C., Haertel, G. D. \& Walberg, H. J. (1993). Toward a Knowledge Base for School Learning. Review of Educational Research, 63 (3), 249-294.

Weinert, F. E. \& Helmke, A. (1996). Der gute Lehrer: Person, Funktion oder Fiktion? Zeitschrift für Pädagogik, Beiheft 34, 223-233.

Schlagworte: Unterricht, Unterrichtsstil, Disziplin, Schülerverhalten, LehrerSchüler-Beziehung, Regel, Regelung, Führung 


\title{
Contenu des règles et gestion de classe
}

\author{
Résumé
}

Certaines règles contribuent de manière importante à un enseignement couronné de succès, à savoir à un enseignement sans trop de dérangements et avec une bonne participation. Jusqu'ici, la recherche ne s'est guère intéressée ni au contenu de ces règles, ni à la relation entre la structure de ces règles et la gestion de classe. Sur la base de données portant sur des maîtres d'école $(\mathrm{N}=605)$ et des élèves $(\mathrm{N}=923)$, le présent article se focalise sur ces deux questions. Il montre les aspects pour lesquels des règles doivent être établies : l'interaction sociale, le silence et l'ordre. Il apparaît également qu'une centration unilatérale sur des règles visant l'ordre entraîne un plus haut niveau de dérangement. Pour finir, le caractère indirect de ce rapport est illustré par un modèle et par une discussion sur le rôle de variables intermittentes, en particulier la relation entre enseignant et élève.

Mots clés: enseignement, style d'enseignement, méthode pédagogique, discipline, relation maître-élève

\section{I contenuti delle regole e della gestione della classe}

\section{Riassunto}

Grazie alla riduzione degli elementi di disturbo e al miglioramento della collaborazione, le regole forniscono un contributo importante all'insegnamento. La ricerca ha però finora trscurato i contenuti delle regole e la relazione esistente tra regole e gestione della classe. Un'inchiesta condotta presso insegnanti $(\mathrm{N}=605)$ e allievi $(\mathrm{N}=923)$ mette in evidenza la necessità delle regole per gli ambiti dell'interazione sociale e di una gestione tranquilla e ordinata. Se per contro ci si concentra unilaterlamente sulle regole, l'effetto e sovente un aumento delle interferenze e dei disturbi. In conclusione l'articolo discute un modello che illustra il carattere indiretto di questa relazione e si analizza il ruole delle variabili intermittenti (in particolare al relazione insegnante-allievo).

Parole chiave: insegnamento, stile d'insegnamento, dissciplina, comportamento degli allievi, relazione insegnante-allievo, regole, conduzione 


\section{The content of rules and classroom management}

\section{Summary}

Rules make an important contribution to successful teaching where there is little disruption and a high level of participation. However, so far research has barely paid attention to the contents of these rules and to their relationship to the classroom management. This article highlights with the help of teachers' $(\mathrm{N}=605)$ and pupils' $(\mathrm{N}=923)$ data these questions. It identifies aspects (social interaction, silence and order) where regulation is needed. But an unbalanced focusing on rules of order is often accompanied by a higher degree of disruption during classes. Finally, the indirect nature of this correlation is illustrated in a model and the role of the intermittent variables (especially the relationship between teachers and pupils) is discussed.

Keywords: teaching, behavior incentive, discipline, teacher-pupil relation 
T h e m a 